\title{
Recovery Strategies Implemented by Sport Support Staff of Elite Rugby Players in South Africa
}

\begin{abstract}
:
Objective: The main aim of this study was to determine strategies used to accelerate recovery of elite rugby players after training and matches, as used by medical support staff of rugby teams in South A frica. A secondary aim was to focus on specifics of implementing ice/cold water immersion as recovery strategy.

Design: A Questionnaire-based cross sectional descriptive survey was used. Setting and Participants: Most ( $n=58)$ of the medical support staff of rugby teams (doctors, physiotherapists, biokineticists and fitness trainers)

Van Wyk DV, BPhyst (UP)'; Lambert MI, MRC/UCT ${ }^{2}$

\author{
${ }^{1}$ Registered Physiotherapist. \\ Medicine, Department of Human Biology, \\ Faculty of Health Sciences, University of \\ Cape Town, South Africa.
}

2 Research Unit for Exercise Science and Sports who attended the inaugural Rugby Medical Association conference linked to the South African Sports Medicine Association Conference in Pretoria (14-16th November, 2007) participated in the study.

Results: Recovery strategies were utilized mostly after matches. Stretching and icelcold water immersion were utilized the most (83\%). More biokineticists and fitness trainers advocated the usage of stretching than their counterparts (medical doctors and physiotherapists). Ice/Cold water immersion and Active Recovery were the top two rated strategies. A summary of the details around implementation of ice/cold water therapy is shown (mean) as utilized by the subjects: (i) The time to immersion after matches was $12 \pm 9 \mathrm{~min}$; (ii) The total duration of one immersion session was $6 \pm 6$ min; (iii) 3 immersion sessions per average training week was utilized by subjects; (iv) The average water temperature was $10 \pm 3{ }^{\circ} \mathrm{C}$.; (v) Ice cubes were used most frequently to cool water for immersion sessions, and (vi) plastic drums were mostly used as the container for water.

Conclusion: In this survey the representative group of support staff provided insight to which strategies are utilized in South African elite rugby teams to accelerate recovery of players after training and/or matches.
\end{abstract}

\section{KEYWORDS: RUGBY, ELITE PERFORMANCE, RECOVERY.}

\section{INTRODUCTION}

Following the introduction of professionalism in rugby in 1995 , the physical demands on the players has increased significantly (Garraway et al, 2000). Training and competition challenges the neurological, physiological, nutritional and psychological functions of the athlete (Calder, 2003). It is known that a rugby match causes structural damage to muscles even in the "uninjured" players (Takarada, 2003). This conclusion was made by measuring plasma creatine kinase activity of rugby players before and after competitive matches and observing that the levels were elevated, indicative of soft tissue injury. The extent of the muscle damage in this study was highly dependant on the number of tackles made or received.
The damage could also have arisen from the repetitive, eccentric muscle actions involved in the intermittent running/ sprinting during rugby matches. This was confirmed in a study by Thompson et al (1999) who studied the effect of eccentric muscle actions during running in rugby, soccer, field hockey and basketball players.

These stressful components of training and competition may thus temporarily impair an athlete's performance (Barnett, 2006). This impairment may range from transitory (lasting minutes or hours after training or competition), to a longer period (several days). Laboratory studies show that the muscles take at least 5 days for the subtle muscle pain to subside and even longer before the functional aspects of the muscles are fully recovered (Cheung et al, 2003). However, professional rugby players often have to play a match every 7 days for several consecutive weeks. This imposes a serious physiological stress as the players have to train and compete while their muscles are in a state of disrepair. Lack of appropriate recovery

\section{Correspondence to: \\ David V Van Wyk \\ PO Box 12000 , \\ Erasmuskloof 0048 \\ Tel: $\quad+27123454802$ \\ Fax: +27123453294}

Email: david_van_wyk@hotmail.com

In partial fulfillment of the MPhil Sports Physiotherapy degree 
may result in the player being unable to train at the required intensity or complete the required load at the next training session or match. This results in a decline in performance and increased risk of injury (Barnett, 2006).

To be able to cope with this adaptive stress, it is very important to recover from training/competition. Various modalities of recovery are utilized in an attempt to restore and adapt in preparation for the next training session/ competition (Barnett, 2006). The ultimate aim of these modalities is to reduce fatigue, injury and the transient impairment in performance while encouraging effective adaptation as noted by Barnett (2006). Equally as important as a warm up routine, is a recovery routine beginning immediately after training/competition (cool down) and continuing for up to 24 hours post training effect or competition (Barnett, 2006).

A review of the literature on the popular strategies which are used to accelerate recovery in rugby (cryotherapy, massage, stretching, compression garments, active recovery, nutrition, sleep and non-steroidal anti-inflammatories) shows no overwhelming evidence supporting any of these techniques (Barnett, 2006). This can be attributed to the fact that many of the studies are laboratory based and the protocols which are used to simulate training or competition may lack specificity with regards to the timing after the event, frequency, duration and intensity. Another potential problem is that the markers which are used to define the state of recovery are indirect and also lack specificity as mentioned earlier.

Whilst it may be concluded, based on anecdotal data, that recovery strategies are effective, there is insufficient data to support their effectiveness in an evidence-based way.

Thus, before the efficacy and mechanism of the recovery procedures could be investigated the author wanted to find out what practitioners associated with rugby teams are actually implementing as recovery strategies.

Therefore the aim of this study was to do a survey in a representative group of support staff (doctors, physiotherapists, biokineticists and fitness trainers) who are involved with rugby teams, to determine which strategies they use to accelerate recovery of the players after training and matches. In particular, details about ice/cold water immersion as a recovery strategy were investigated. This information may serve to inform studies investigating the efficacy and mechanism of actions of the various recovery procedures.

\section{METHODS}

\section{Study design}

This study was a questionnaire-based cross sectional descriptive survey.

\section{Subjects and testing procedure}

All the medical support staff of rugby teams (doctors, physiotherapist, biokineticists and fitness trainers) who attended the inaugural Rugby Medical Association conference linked to the South African Sports Medicine Association Conference in Pretoria (14-16th November, 2007) were invited to participate in the study. All participants ( $\mathrm{n}=$ 58) were given information about the study, and assured that their names would not be linked to the data. They also had the option of withdrawing from the study at any time. They signed an informed consent form before completing a questionnaire. All the data were therefore collected in such a manner that individual subjects were not identifiable, and confidentiality was at all times maintained. The study was approved by the Research and Ethics Committee of the Faculty of Health Sciences, University of Cape Town.

\section{Questionnaire}

The questionnaire used for the survey was developed from the review paper on recovery strategies by Barnett (2006). In a pilot study towards content validity for the drafted questionnaire, a sample of health professionals, who were similarly qualified to the health professionals targeted in this study, completed the first draft of the questionnaire. Any ambiguous questions were adjusted. The revised questionnaire was tested again on a different sample of health professionals, and questions were adjusted where appropriate.

\section{Data analysis}

The answers to the questions were transcribed into a spreadsheet and checked for transcribing errors before analysis.

\section{Statistics}

The descriptive data are presented as means and standard deviations or percentages. There was concern about lack of statistical power when the groups were subdivided hence we avoided using comparative statistics.

\section{RESULTS AND DISCUSSION}

\section{Subjects}

The first finding of this study was that subjects associated with sport team structures were highly experienced in the usage of recovery strategies. The majority (48\%) of the subjects had been using recovery strategies for $0-5$ years. Generally subjects agreed that the planning and executing of recovery must follow a multi-disciplinary approach. In summary, it can be concluded that the group represented practitioners who were dealing with issues around recovery of rugby players on almost a daily basis and therefore their responses to the questions should be interpreted in this context. Due to the novelty of this survey, no other data could be found to compare with the above findings.

\section{When strategies are utilized}

Overall, based on the means of all match and training situations, recovery strategies were mostly used after matches (33\%), followed by after training sessions. Table 1 will show the various training and match situations and their association with recovery strategies.

\section{Types of recovery strategies used}

The second important finding of this study was that the most prevalent recovery modalities reported by the sport support staff are stretching and ice/cold water immersion. This is followed by active recovery and massage. A breakdown of all the recovery strategies 
Table 1. Various training and match situations and their association with recovery strategies. The values represent a percentage of the number of respondents $(n=58)$.

\begin{tabular}{|l|c|c|c|c|c|c|}
\hline STRATEGY & $\underline{\mathbf{1}}$ & $\underline{\mathbf{2}}$ & $\underline{\mathbf{3}}$ & $\underline{\mathbf{4}}$ & $\underline{\mathbf{5}}$ & $\underline{\mathbf{6}}$ \\
\hline STRETCHING & $\underline{\mathbf{7 4}}$ & $\underline{5}$ & $\underline{52}$ & $\underline{5}$ & $\underline{16}$ & $\underline{29}$ \\
\hline COLD/ICE WATER IMMERSION & $\underline{28}$ & $\underline{41}$ & $\underline{\mathbf{6 7}}$ & $\underline{12}$ & $\underline{14}$ & $\underline{26}$ \\
\hline ACTIVE RECOVERY & $\underline{2}$ & $\underline{22}$ & $\underline{47}$ & $\underline{10}$ & $\underline{16}$ & $\underline{24}$ \\
\hline MASSAGE & $\underline{5}$ & $\underline{17}$ & $\underline{33}$ & $\underline{\mathbf{1 4}}$ & $\underline{\mathbf{2}}$ & $\underline{43}$ \\
\hline ADDITIONAL HYDRATION & $\underline{60}$ & $\underline{10}$ & $\underline{59}$ & $\underline{3}$ & $\underline{7}$ & $\underline{10}$ \\
\hline NUTRITION: EXTRA CARBOHYDRATES & $\underline{45}$ & $\underline{17}$ & $\underline{55}$ & $\underline{3}$ & $\underline{5}$ & $\underline{3}$ \\
\hline COMPRESSION GARMENTS & $\underline{17}$ & $\underline{12}$ & $\underline{22}$ & $\underline{7}$ & $\underline{7}$ & $\underline{36}$ \\
\hline LIMITING/AVOIDING ALCOHOL USE & $\underline{47}$ & $\underline{9}$ & $\underline{64}$ & $\underline{5}$ & $\underline{17}$ & $\underline{29}$ \\
\hline NUTRITION:EXTRA PROTEIN & $\underline{36}$ & $\underline{16}$ & $\underline{45}$ & $\underline{5}$ & $\underline{7}$ & $\underline{9}$ \\
\hline NUTRITION: EXTRA ELECTROLYTES & $\underline{48}$ & $\underline{5}$ & $\underline{48}$ & $\underline{9}$ & $\underline{10}$ & $\underline{10}$ \\
\hline NSAIDS & $\underline{0}$ & $\underline{3}$ & $\underline{3}$ & $\underline{7}$ & $\underline{10}$ & $\underline{45}$ \\
\hline ADDITIONAL SLEEP AND REST & $\underline{7}$ & $\underline{16}$ & $\underline{22}$ & $\underline{\mathbf{1 4}}$ & $\underline{\mathbf{2 9}}$ & $\underline{17}$ \\
\hline CONTRAST TEMPERATURE WATER IMMERSION & $\underline{12}$ & $\underline{12}$ & $\underline{29}$ & $\underline{2}$ & $\underline{5}$ & $\underline{10}$ \\
\hline ELECTROMYOSTIMULATION & $\underline{0}$ & $\underline{3}$ & $\underline{0}$ & $\underline{3}$ & $\underline{5}$ & $\underline{17}$ \\
\hline OTHER STRATEGIES & $\underline{3}$ & $\underline{2}$ & $\underline{5}$ & $\underline{3}$ & $\underline{2}$ & $\underline{0}$ \\
\hline VISUALISATION & $\underline{2}$ & $\underline{0}$ & $\underline{2}$ & $\underline{3}$ & $\underline{2}$ & $\underline{0}$ \\
\hline HYPERBARIC OXYGEN & $\underline{0}$ & $\underline{0}$ & $\underline{0}$ & $\underline{0}$ & $\underline{0}$ & $\underline{0}$ \\
\hline MEAN & $\underline{24}$ & $\underline{11}$ & $\underline{\mathbf{3 3}}$ & $\underline{6}$ & $\underline{11}$ & $\underline{18}$ \\
\hline
\end{tabular}

KEY:
1-AFTER EVERY SINGLE TRAINING SESSION,
4- ONLY IF MATCH WAS HARD,
2-ONLY IF TRAINING WAS HARD,
3- AFTER EVERY MATCH,
5- IF PLAYER IS OVERTRAINED,
6- IF PLAYER IS INJURED

is shown in Table 2. The top 3 utilised recovery strategies will be highlighted.

\section{Stretching}

The main goal of stretching is proposed to increase the range of motion about joints and the data showing that this indeed occurs is convincing (Beaulieu, 1991; Thacker et al, 2004). Stretching is also commonly advocated as a technique for reducing the risk of injury (Hartig et al, 1999) although the research does not support this (Thacker et al, 2004; Anderson, 2005). Although stretching was a very popular recovery strategy reported in this survey, the evidence supporting stretching as part of a recovery protocol is not convincing. Stretching was the strategy mostly reported to be used after every single training session $(74 \%)$, followed by usage after every match (52\%). Stretching was also rated as an "effective" strategy by the 58 subjects in this survey. All of the biokineticists (100\%) and fitness coaches $(100 \%)$ advocated the usage of stretch-
Table 2: Recovery strategies used by respondents arranged in order of use $(n=58)$

\begin{tabular}{|l|l|}
\hline STRATEGIES & PERCENTAGE \\
\hline STRETCHING & $\underline{\mathbf{8 3}}$ \\
\hline ICE/COLD WATER IMMERSION & $\underline{\mathbf{8 3}}$ \\
\hline ACTIVE RECOVERY & 74 \\
\hline MASSAGE & 66 \\
\hline ADDITIONAL HYDRATION & 59 \\
\hline NUTRITION: EXTRA CARBOHYDRATES & 54 \\
\hline COMPRESSION GARMENTS & 53 \\
\hline LIMITING/AVOIDING ALCOHOL USE & 49 \\
\hline NUTRITION: EXTRA PROTEIN & 49 \\
\hline NUTRITION: EXTRA ELECTROLYTES & 48 \\
\hline NSAIDS & 37 \\
\hline ADDITIONAL SLEEP AND REST & 36 \\
\hline CONTRAST TEMPERATURE WATER IMMERSION & 27 \\
\hline ELECTROMYOSTIMULATION & 14 \\
\hline OTHER STRATEGIES & 10 \\
\hline VISUALIZATION & 7 \\
\hline HYPERBARIC OXYGEN & 0 \\
\hline
\end{tabular}


ing, followed by physiotherapists $(89 \%)$ and doctors $(71 \%)$. While a mechanism by which stretching may enhance the recovery process has yet to be identified, it has been suggested that stretching may disperse oedema accumulated during tissue damage (Bobbert et al, 1986). This theory however has not been proved. Furthermore, there do not appear to be any studies that have investigated the effect of stretching between exercise sessions/matches on performance during post-recovery exercise/ competition (Barnett, 2006). Stretching exercises have been shown to be ineffective in reducing the symptoms of muscle damage (Cheung et al, 2003; Connolly et al, 2003; Gulick et al, 1996; Mika et al, 2007). A comprehensive review of studies which had used stretching after exercise (total stretching time ranging from 300 to 600 seconds) with the goal of reducing muscle soreness, showed that 72 hours after exercise, pain had only reduced by $2 \%$ which was not regarded as meaningful (Anderson, 2005).

\section{Ice/cold water immersion}

Various forms of cryotherapy are used as an intervention for post exercise recovery, particularly after exercise which raises body temperature, and causes inflammation of muscles (Smith, 1991). Of these forms ice/cold water immersion is probably the most popular. The basis for using ice/cold water immersion is on the assumption that it is effective for decreasing metabolic rate, inflammation, blood flow, and skin muscle and intra-articular temperatures, thus accelerating recovery after training/ competition (Merrick et al, 1991). Forty eight out of 58 subjects in this survey indicated that they use ice/cold water immersion as a recovery strategy (83\%). Biokineticists were represented the most in this group, followed by physiotherapists, fitness coaches and doctors. Ice/cold water immersion was reported to achieve the highest overall efficacy rating as recovery strategy in this survey with $34 \%$ of subjects rating it as "effective" and $36 \%$ of subjects rating it “extremely effective'. Ninety-six percent of subjects reported utilizing ice/old water immersion after matches and 58\% after training.

It is noted that the medical staff in this survey value ice/cold water immersion as a very important strategy for recovery after matches and/or training. Therefore different studies about ice/ cold water immersion will be reviewed and compared to results found in this survey.

To avoid confusion, the following explanation regarding ice/cold water immersion is included:

Total duration of one immersion session $=x$ amount of single immersions

In order to identify an effective ice/cold water immersion protocol to be subjected to scientific scrutiny, it is necessary to calculate optimum parameters of ice/cold water immersion. Six prominent studies were selected and compared with the mean parameters of this survey after matches (Table 3). Thereafter a mean was calculated for all the parameters in the search for an effective protocol.

When comparing different studies a number of factors need to be controlled, such as the intervention causing the fatigue and muscle damage, the type of cold water immersion protocol, the duration of exposure, the body area immersed and the immersion temperature. Given these limitations, the available data are rather sparse making it difficult to formulate an evidence-based decision about the efficacy of cold water immersion after rugby. There is contra-

Table 3: Parameters for ice/cold water immersion in the current literature compared to this survey.

\begin{tabular}{|c|c|c|c|c|c|c|c|c|}
\hline PARAMETER & $\begin{array}{l}\text { This } \\
\text { Survey } \\
\text { (mean) } \\
(n=43)\end{array}$ & $\begin{array}{l}\text { Eston } \\
\text { et al } \\
(1999) \\
(n=15)\end{array}$ & $\begin{array}{l}\text { Sellwood } \\
\text { et al } \\
(2007) \\
(n=40)\end{array}$ & $\begin{array}{l}\text { Vaille } \\
\text { et al } \\
(2008) \\
(n=10)\end{array}$ & $\begin{array}{l}\text { Bailey } \\
\text { et al } \\
(2007) \\
(n=20)\end{array}$ & $\begin{array}{l}\text { Burke } \\
\text { et al } \\
(2001) \\
(n=45)\end{array}$ & $\begin{array}{l}\text { Paddon- } \\
\text { Jones } \\
\text { et al } \\
(1997) \\
(n=8)\end{array}$ & Mean \\
\hline $\begin{array}{l}\text { Time to immersion } \\
\text { (min) }\end{array}$ & $12 \pm 9$ & Immed & Immed & 5 & Immed & Immed & Immed & - \\
\hline Total duration (min) & $6 \pm 6$ & 15 & 3 & 5 & 10 & 10 & 20 & 10 \\
\hline $\begin{array}{l}\text { Duration single } \\
\text { immersion (min) }\end{array}$ & $3 \pm 3$ & 15 & 1 & 1 & 10 & 10 & 20 & 9 \\
\hline $\begin{array}{l}\text { Consecutive } \\
\text { immersions }\end{array}$ & $2 \pm 1$ & 1 & 3 & 5 & 1 & 1 & 1 & 2 \\
\hline $\begin{array}{l}\text { Immersion } \\
\text { sessions pw }\end{array}$ & 3 & 3.5 & 1 & 1 & 1 & 5 & 1 & 2 \\
\hline Temp of water $\left(\mathrm{C}^{\circ}\right)$ & $10 \pm 3$ & 15 & 5 & $10 / 15 / 20$ & 10 & 8 & 5 & 10 \\
\hline How water is cooled & $\begin{array}{l}\text { Ice } \\
\text { cubes }\end{array}$ & N.A & $\begin{array}{l}\text { Melting } \\
\text { iced } \\
\text { water }\end{array}$ & N.A & $\begin{array}{l}\text { Crushed } \\
\text { Ice }\end{array}$ & N.A & Ice & - \\
\hline Type of container & $\begin{array}{l}\text { Plastic } \\
\text { drum }\end{array}$ & N.A & N.A & $\begin{array}{l}\text { Inflatable } \\
\text { bath }\end{array}$ & bath & N.A & $\begin{array}{l}\text { Plastic } \\
\text { cooler }\end{array}$ & - \\
\hline
\end{tabular}


dicting evidence for the use of ice/cold water immersion (Yamane et al, 2006). However, physiological evidence aside, there does seem to be anecdotal evidence for using cold water immersion after a match or hard training session. Perhaps the ritual of undergoing the cold water therapy focuses attention on recovery, and the increased attention indirectly enhances the recovery process? Further research is needed to answer this question.

\section{Active Recovery}

Despite the lack of understanding of the mechanisms of active recovery, there are several studies which show that this method has some positive effects. For example, a recent study on rugby players demonstrated that recovery rates (using creatine kinase in transdermal exudate as a marker) were similar for active recovery, contrast temperature water immersion and wearing lower body compression garments, and were significantly better than passive recovery (Gill et al, 2006). Another study also showed that after high-intensity training a 15 minute treatment of either active recovery (cycling at $30 \% \quad \mathrm{VO}_{2} \mathrm{max}$ ) and massage, or cold water immersion $\left(15^{\circ} \mathrm{C}\right)$ improved recovery (measured by work performed) in contrast to a group which did not use any of these strategies (Lane et al, 2004). Active recovery enhances the removal of high levels of circulating lactate (Brooks et al, 2005). However, this beneficial physiological effect is not reported by all studies. Suzuki et al (2004) found that active recovery did not have any effect on recovery (measured by circulating creatine kinase and neutrophils) after a rugby match, although the players seemed to have better mental recovery after being exposed to an active recovery protocol consisting of low intensity exercise (Suzuki et al, 2004).

In this survey, active recovery was reported to be used by $74 \%$ of subjects, mostly after matches. It was also rated as an "effective" strategy by most subjects and reported to be used firstly by biokineticists, followed by fitness coaches, physiotherapists and doctors.

A possible future recommendation is for surveys to evaluate the specific type of active recovery, as there is many to choose from. The variety of protocols could also lead to different effects on the recovery process.

\section{CONCLUSION}

Recovery modalities are gaining wide acceptance among elite athletes and especially professional rugby players. Sporting bodies are investing time and money in providing these modalities and therefore, further research and better consideration of the evidence of their effectiveness appear warranted.

A review of the studies shows clearly that there are varied responses to all the recovery strategies. This variation can be attributed to the different experimental protocols for inducing fatigue/muscle damage and the outcome measures which are indirect markers of recovery. Many aspects of recovery are very difficult to measure in the laboratory and the indirect markers of various aspects of recovery lack the required precision to detect small, yet meaningful changes. Where does that leave the practitioner, driven by the desire to have an evidencebased approach to the management of the players? It would be foolish to conclude that recovery strategies do not have an affect, although a strict analysis of the studies might lead one to this conclusion. While there is only anecdotal evidence which has "stood the test of time" and which suggests that there is a role for recovery strategies after training and competition. Whilst the specific details are not known, a logical interpretation of the available knowledge suggests that under certain circumstances there is a role for cryotherapy(ice/cold water immersion), active recovery, nutrition, compression garments, massage, stretching and "power" naps as part of a recovery strategy. The studies which have examined a combination of the strategies together as shown by Tessitore et al (2007) and Dawson et al (2005), suggest that "something is better than nothing", so the details of the followed protocol should be custom-made based on the circumstances and equipment available to the support staff and team. It has been suggested that the recovery strategies should follow a pattern and become a habit (Jeffreys, 2007). Prior to embarking on a strategy for recovery, it is important that the rugby players are educated about the process so that they are fully informed about the protocol and assume some personal responsibility. It is important that within a team setting the recovery strategies adopted by the support staff become routine and almost ritualistic (Jeffreys, 2005). It is also prudent to individualize the recovery strategies for various players. For example, older players with a history of joint injuries might be handled differently to younger players. Also, players of different positions, who are faced with different physical demands, might also have different strategies after a match (Duthie et al, 2003; Scott et al, 2004). The strategies which are going to be used should be customized for each player, discussed with the player and then implemented in a systematic way. Ambitious strategies which cannot be used when the team is traveling should be avoided as this may cause psychological problems with the players when they cannot use a procedure which they are accustomed to.

In the ongoing search for an optimum recovery protocol that could be subjected to scientific scrutiny, the above recommendations made by the authors could be used for future scientific testing.

For future purposes other surveys could be aimed at the different levels of sport as well as the different types of sport, especially high intensity sports such as soccer, field hockey and basketball.

\section{REFERENCES}

Bailey DM, Erith SJ, Griffin PJ, Dowson A, Brewer DS, Gant N and Williams C 2007 Influence of cold-water immersion on indices of muscle damage following prolonged intermittent shuttle running. Journal of Sports Sciences 25:1163-1170

Barnett A 2006 Using recovery modalities between training sessions in elite athletes. Does it help? Sports Medicine 36: 781-796

Burke DG, Holt LE, Rasmussen R, Mackinnon NC, Vossen JF and Pelham TW 2001 Effects of hot or cold water immersion and modified proprioceptive neuromuscular facilitation flexibility exercise on hamstring length. Journal of Athletic Training 36:16-19 
Cheung K, Hume PA and Maxwell L 2003 Delayed onset muscle soreness: treatment strategies and performance factors. Sports Medicine 33:145-64

Connolly DA, Sayers SP and Mchugh MP 2003 Treatment and prevention of delayed onset muscle soreness. Journal of Strength and Conditioning Res 17:197-208

Eston R and Peters D 1999 Effects of cold water immersion on the symptoms of exercise-induced muscle damage. Journal of Sports Science 17:231-8

Gill ND, Beaven CM and Cook C 2006 Effectiveness of post-match recovery strategies in rugby players. British Journal of Sports Medicine 40:260-263

Hartig DE, Fredriksson K and Perrson I 1999 Increasing hamstring flexibility decreases lower extremity overuse injuries in military basic trainees. American Journal of Sports Medicine 27:173-176
Hemmings B, Smith $\mathrm{M}$ and Grayden $\mathrm{J}$ et al 2000 Effects of massage on physiological restoration, perceived recovery, and repeated sports performance. British Journal of Sports Medicine 34:109-15

Jeffreys I 2007 Post game recovery strategies for rugby. NSCA's Performance Training Journal 6:13-16

Lane KN and Wenger HA 2004 Effect of selected recovery conditions on performance of repeated bouts of intermittent cycling separated by 24 hours. Journal of Strength and Conditioning Res 18:855-60

Paddon-Jones DJ and Quigley BM 1997 Effect of cryotherapy on muscle soreness and strength following eccentric exercise. International Journal of Sports Medicine 18:588-593

Sellwood KL, Brukner P, Williams D, Nicol A and Hinman R 2007 Ice water immersion and delayed onset muscle soreness: a randomised controlled trial. British Journal of Sports Medicine 000:1-7

Suzuki M, Umeda T, Nakaji S, Shimoyama T, Mashiko T and Sugawara K 2004 Effect of incorporating low intensity exercise into the recovery period after a rugby match. British Journal of Sports Medicine 38:436-440

Vaile J, Halson S, Gill N and Dawson B 2008 Effect of cold water immersion on repeat cycling performance and thermoregulation in the heat. Journal of Sport Sciences 26:431-440

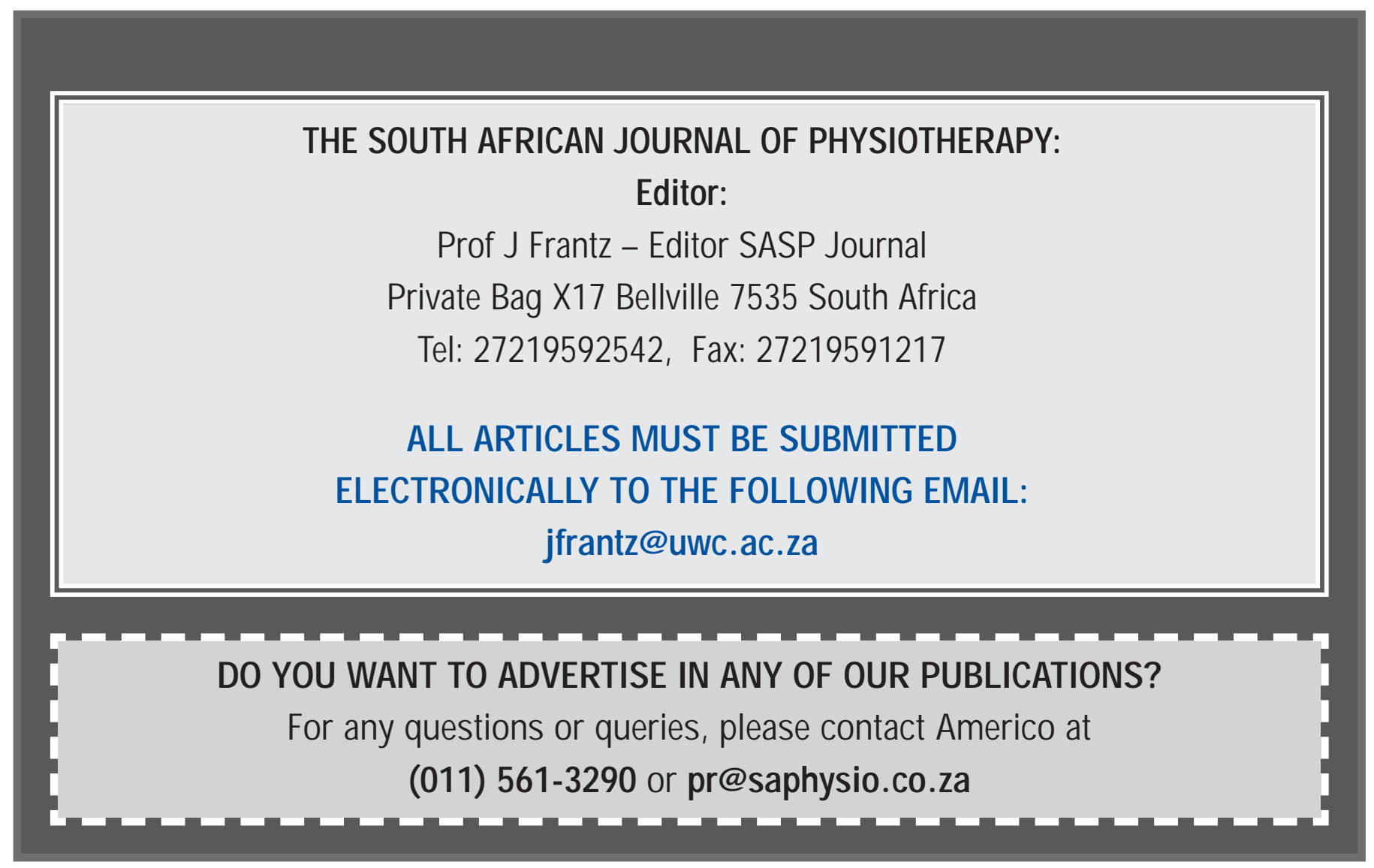

